CASE REPORT

\title{
Association of Vogt Koyanagi Harada Syndrome and Seronegative Rheumatoid Arthritis
}

\author{
Teoman Aydin ${ }^{1}$, Ozgur Taspinar ${ }^{2}$, Meryem Guneser ${ }^{1}$, Yasar Keskin ${ }^{1}$
}

\begin{abstract}
BACKGROUND: Vogt Koyanagi Harada (VKH) Syndrome is a rarely-seen multi-systemic, autoimmune and inflammatory disease. It observed frequently with neurologic, auditory and skin manifestations and characterized with bilateral, chronic and diffused granulomatous panuveitis. It generally affects women in young-adult period.

CASE: A 57 year-old female patient applied to a special center one year ago with a complaint of decrease in the sight acuity of the right eye. The right eye was operated on with cataract diagnosis. Uveitis was developed firstly in the right eye and then in the left eye after the operation. Having complaints about uveitis, tinnitus and hear loss, the patient was diagnosed with VKH syndrome. The pains started to be felt in small hand joints and both of the two ankles. The pains were increasing especially in the mornings and during rest. The duration of morning stiffness was two hours in hand and foot joints. The patient had had lumbar pain with mechanic characteristic for five years.

CONCLUSION: Being diagnosed with seronegative rheumatoid arthritis (RA), our case is presented because VKH syndrome is rarely seen in Turkey, and the joint findings are at the forefront.

Keyword: Seronegative Rheumatoid Arthritis, joint involvement, Vogt Koyanagi Harada Syndrome
\end{abstract}

DOI: http://dx.doi.org/10.4314/ejhs.v26i2.14

\section{INTRODUCTION}

Vogt Koyanagi Harada Syndrome is a disease with chronic course characterized with neurologic and skin symptoms besides the bilateral granulomatous pan uveitis (1). Although the etiology of the disease is not known exactly, it is thought to be caused by autoimmunity created against antigen controlled via T-lymphocytes and associated with melanocytes (2). VKH disease is more prevalent in certain racial and ethnic groups, particularly in Asian individuals and certain LatinAmerican groups. And individuals have certain HLA groups in Japan $(3,4)$.

Vogt Koyanagi Harada Syndrome diagnosis is based on making distinguishing it from other uveitis reasons with clinic and laboratory findings (5). Besides neurologic and skin symptoms, the disease consists of typical bilateral exudative retina decollement in eyes, palillitis, choroiditis and iridocyclitis. In this article, the clinical course of a VKH case with delayed diagnosis having ocular and systemic findings suppressed due to corticosteroid treatment is presented. Rheumatoid arthritis is an inflammatory disease having chronic systemic process. Its etiology is not nown properly, but it is also known that it generally affects the joints. On the other hand, it has a significant extra-articular involvement with nearly all organ systems influenced. The prevalence in women is three times more than that in men. It has been proved that there is a genetic component transferred through HL A-DQ and-DR alleles (6).

Being diagnosed with seronegative rheumatoid arthritis diagnosis, our case is our case is presented because VKH syndrome is rarely seen in Turkey. No joint findings are seen inVKH syndrome.

\footnotetext{
${ }^{1}$ Bezm-i Alem Foundation University School Of Medicine, Department Of Physical Therapy And Rehabilitation, Istanbul.

${ }^{2}$ Cinarcik State Hospital, Department Of Physical Therapy And Rehabilitation, Yalova

Corresponding Author: Ozgur Taspinar, Email: drozgur82@gmail.com
} 


\section{CASE}

A 57 year-old female patient applied to a special center one year ago with a complaint of decrease in the sight acuity of the right eye. The right eye was operated on due to cataract diagnosis. Uveitis was developed firstly in the right eye and then in the left eye after the operation. The patient was suit for the VKH syndrome because of the ophthalmologists' opinion, patients' age, and skin and auditory involvements. She patient was diagnosed VKH syndrome according to uveitis, tinnitus and hearing sense loss complaints. She had joint complaints and complained of joint problems for more than six weeks. That's why; she was examined. The pains started to be felt in small hand joints and both of the two ankles. The pains were increasing especially in the mornings and during rest. The duration of morning stiffness was two hours in hand and foot joints. The patient had had lumbar pain with mechanic characteristic for five years. There was not any pain during nights. She had given birth to two children. Considering her family background, it was learnt that her maternal twin had psoriasis. There was not any rheumatic disease in family her background. No psoriasis was detected in the patient.

In physical examination, there was redness in the cornea of left eye and the $3 \mathrm{~cm}$ alopecic region in hairy skin. There were pressure-induced pains in proximal interphalangeal joints of both hands, metacarpaphalangeal joints of the $4^{\text {th }}$ and $5^{\text {th }}$ fingers in the left hand, wrists, elbows, and both of the two achilles tendons. There was swelling in the $4^{\text {th }}$ and $5^{\text {th }}$ metacarpaphalangeal joints of the left hand. Lumbar region, hip and sacroiliac joint examinations were normal. In neurologic examination, bilateral visual acuity and hear loss were identified.

In laboratory evaluation, the hemogram values were normal, sedimentation was $36 \mathrm{~mm} / \mathrm{h}$ and CRP was $1.0 \mathrm{mg} / \mathrm{dl}$; rheumatoid factor, antinuclear antibody, anti ds-DNA, anti-CCP, and HLA B27 tests were negative. There were erosions in the PIF joints in the hand radiography of patient (Figure 1). In foot magnetic resonance imaging, synovitis was observed (Figure 2).
Sacroiliac joint magnetic resonance imaging was normal. With those findings, the patient diagnosed with Seronegative Rheumatoid Arthritis diagnosis, and the consensus was reached with ophthalmic polyclinic about VKH and Seronegative Rheumatoid Arthritis association. The patient was taken to follow-up with metothrexate $10 \mathrm{mg} /$ week, prednisolone $5 \mathrm{mg} /$ day treatment schedule.

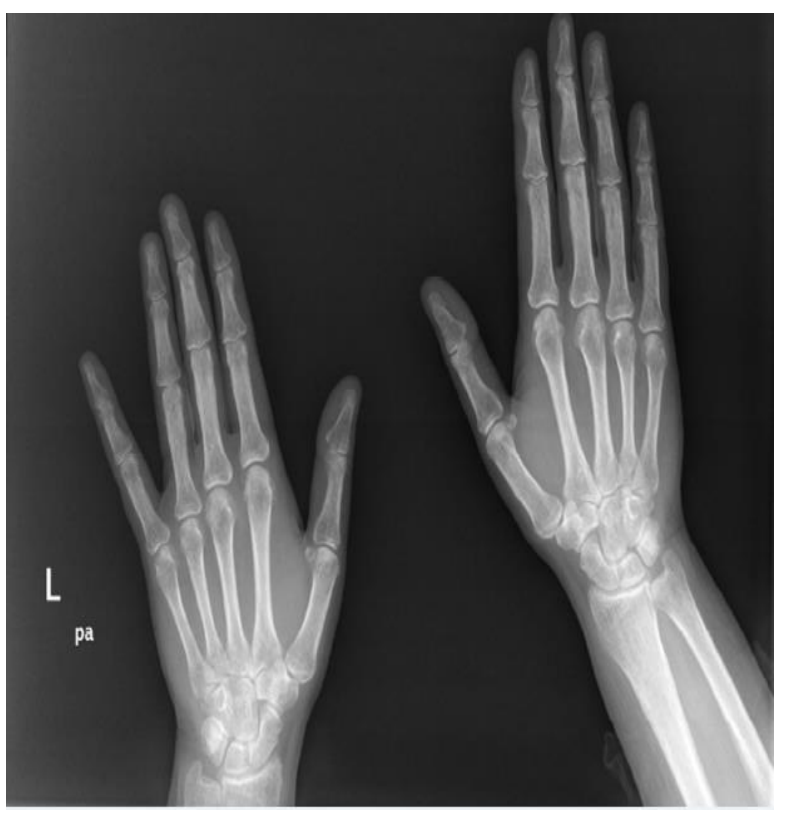

Figure 1: Images of erosion in PIF joints

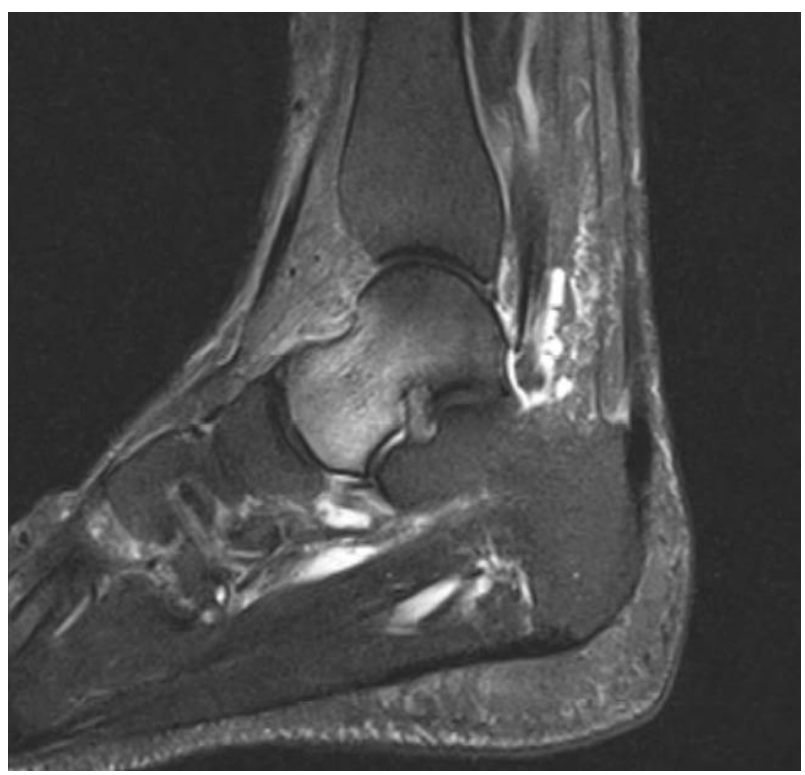

Figure 2: Images of synovitis in foot $M R$ 


\section{DISCUSSION}

Vogt Koyanagi Harada Syndrome is a multisystemic disease characterized with granulomatous inflammation, and affecting the auditory system, the meninx and the skin (1). Despite that, it is rarely seen, has chronic course and the potential of leading to blindness (4). Although its prevalence in our country is not known, it has been reported that it constitutes $10 \%$ of uveitis in Japan (7), and its annual prevalence is 15.5 per million (8).

Vogt Koyanagi Harada Syndrome is generally seen in young middle aged individuals, and more frequently in women (8). Complaints may vary depending on the phase in which the disease is diagnosed. Patients having autoimmune vestibulitis apply with tinnitus complaint, while patients with meninx involvement apply with inflammation, nausea and severe headache. Patients with skin involvement have hypopigmentations around the hands and the face $(1,5$, 8 ). Besides all of those findings, while wide choroid inflammation, exudative retina decollement and papillitis accompany the early stages of this disease, some ocular findings such as coroidal depigmentation are observed in late stages (9). Especially, because it develops bilateral granulomatous uveitis, although it can be confused with sympathetic ophthalmia, it is distinguished from that disease since it does not have VKH trauma background (10).

Vogt Koyanagi Harada syndrome constitutes $8 \%$ of endogen and uveitis in Japan and $1-4 \%$ in uveitis in mixed races in USA. It has been reported in a study that extra ocular findings in VKH syndrome vary depending on races and ethnic groups. While the prevalence values of meningismus, dysacousia, alopecia, polisis and vitilligo are $80 \%, 60 \%, 60 \%$ and $25 \%$ respectively, dermatological findings have been observed much less frequently. Although the first symptoms of the disease appear generally between the ages of $20^{t}$ and 50, it has been reported in a 4 years old child. In genetic factor researches, its relationship with HLADR4 and DW53 has been determined (11). It is assumed that a T-cellmediated autoimmune reaction is created against common membrane antigen in melanocyte and/or neural crest originated tissues (5). This autoimmune response generally results especially with collapse of melanocytes in epidermis, cochlea, meninx and uvea (12).

Our case is a VKH syndrome where the joint involvement is at the forefront. The patient complained of joint problems over than six weeks. The case had joint involvement and morning stiffness in her little joints in the hands and the feet. She had no inflammatory back pain. There was erosions in PIF joints in the hand radiography of the patient (Figure 1). In foot magnetic resonance imaging, synovitis was identified (Figure 2). Sacroiliac joint magnetic resonance imaging was normal. With those findings, the patient was diagnosed with seronegative rheumatoid arthritis diagnosis. Although generally steroid is used in the treatment of VKH syndrome, methotrexate is added into the treatment because there are arthritic findings.

As a result, VKH syndrome is a multi-system syndrome. The system involvement shows variation depending on geographic regions and ethnic origin. Also, it is interesting that both of the two diseases have relationship with HLA genes. With these results, it is a matter of debate provided that the VKH syndrome of our case is joint involvement or seronegative RA.

\section{REFERENCES}

1. Read RW, Rechodouni A, Butani N, et al.: Complications and prognostic factors in VogtKoyanagi-Harada disease. Am J Ophthalmol. 2001;131:599-606.

2. Norose K, Yano A.: Melanoma specific Th1 cytotoxic $\mathrm{T}$ lymphocyte lines in VogtKoyanagi-Harada disease. $\mathrm{Br} J$ Ophthalmol. 1996;80:1002-1008.

3. Snyder DA, Tessler HH. Vogt-KoyanagiHarada syndrome. Am J Ophthalmol 1980;90:69-75.

4. 4.Numaga J, Matsuki K, Tokunaga K, et al.: Analysis of human leukocyte antigen HLADR beta amino acid sequence in VogtKoyanagi-Harada syndrome. Invest Ophthalmol Vis Sci. 1991;32:1958-1961.

5. Read RW, Holland GN, Rao NA, et al.: Revised diagnostic criteria for VogtKoyanagi-Harada disease: report of an international committee on nomenclature. $A m$ J Ophthalmol. 2001;131:647-52. 
6. Aletaha D, Neogi T, Silman AJ, et al: 2010 Rheumatoid arthritis classification criteria: An American College of Rheumatology/ European League Against Rheumatism collaborative initiative. Arthritis Rheum 2010; 62(9):2569-81.

7. Wakabayashi T, Morimura Y, Miyamoto Y, et al.: Changing patterns of intraocular inflammatory disease in Japan. Ocul Immunol Inflamm. 2003;11:277-86.

8. Murakami S, Inaba Y, Mochizuki M, et al.: A nationwide survey on the occurrence of VogtKoyanagi-Harada Disease in Japan. Jpn $J$ Ophthalmol. 1994;38:208-213.
9. Moorthy RS, Inomata H, Rao NA.: VogtKoyanagi-Harada syndrome. Surv Ophthalmol. 1995;39:265-92.

10. Boyd SR, Young S, Lightman S.: Immunopathology of the noninfectious posterior and intermediate uveitides. Surv Ophthalmol. 2001;46:209-233.

11. Read RW.: Vogt-Koyanagi-Harada disease. Ophthalmol Clin North Am 15:333-41,2002

12. Malik S, Gupta AK, Joshi SM, Purohit SN, Khopkar US. Vogt Koyanagi Harada syndrome. Indian Pediatr. 1997;34:1124-6. 